\title{
Analysis and design of almost-periodic vertical-grating-assisted codirectional coupler filters with nonuniform duty ratios
}

\author{
Gia-Wei Chern and Lon. A. Wang
}

\begin{abstract}
A systematic approach to the analysis of almost-periodic vertical-grating-assisted codirectional couplers with nonuniform duty ratios is presented. The Poisson sum formula is used to expand rigorously a nonuniform rectangular grating into a quasi-Fourier series, and local grating parameters such as period, width, and duty ratio can be defined unambiguously in such a procedure. On the basis of this expansion the coupled-local-mode formulation is the most natural extension for the analysis of such a nonuniform grating-assisted codirectional coupler filter. By transformation of the coupled-local-mode equations into the Zakharov-Shabat system the Gel'fand-Levintan-Marchenko inverse-scattering method is then used to synthesize special grating-assisted codirectional coupler filters. The design is illustrated by two typical examples: One is a third-order Butterworth filter, and the other is a linear filter. (C) 2000 Optical Society of America

OCIS codes: $\quad 050.2770,130.2790,230.7400$.
\end{abstract}

\section{Introduction}

Light-wave communication networks such as wavelength-division-multiplexing systems require narrow-band optical filters to achieve hightransmission capacities. One such optical filter is based on the grating-assisted codirectional coupler (GACC), which has attracted much attention; for example, the GACC has been proposed for applications such as wavelength-selective filters, noise filters, tunable filters, and wavelength-selective photodetectors. ${ }^{1-5}$

A vertical GACC is shown schematically in Fig. 1(a). It consists of two stacked slab waveguides with the grating layer located on either slab. Power is exchanged between the two waveguides through the evanescent fields of the two guided modes. For a uniform grating the filter's spectrum is similar to a sinc function and has high sidelobes. These sidelobes can be suppressed with a weighted coupling

The authors are with the Department of Electrical Engineering and Institute of Electro-Optical Engineering, National Taiwan University, Taipei, Taiwan. L. A. Wang's e-mail address is lon@ccms.ntu.edu.tw.

Received 24 January 2000; revised manuscript received 17 May 2000.

0003-6935/00/254629-09\$15.00/0

(C) 2000 Optical Society of America coefficient, ${ }^{6}$ and it was proposed and demonstrated that nonuniform coupling coefficients can be realized by variation of the duty ratio of the rectangular grating. ${ }^{5,7}$ In these studies, conventional apodization functions such as the Gaussian or the Hamming functions were used for weighting the coupling coefficients, and the purpose was to reduce the filter sidelobes. The grating was further divided into several uniform sections, and a fundamental matrixmultiplication method was used to analyze the spectral response. ${ }^{8}$

With the well-developed synthesis methods, ${ }^{9,10}$ one can design a GACC to have a specified filter response by appropriately varying the coupling coefficient and the grating period. The Gel'fandLevintan-Marchenko (GLM) inverse-scattering method was used by Winick ${ }^{11}$ to design a GACC with a third-order Butterworth filter response. However, the geometry considered by Winick was a pair of parallel-channel waveguides embedded in a planar substrate with the grating region located between the two channels. The nonuniform coupling coefficient was realized by variation of the width of the grating with fixed duty ratios. ${ }^{11}$

In this paper a GACC filter with a vertical-coupling geometry [Fig. 1(a)] is our main concern. It has the advantage that the device can be combined with other photonic elements such as photodetectors and quantum-well lasers in a monolithic way. We 


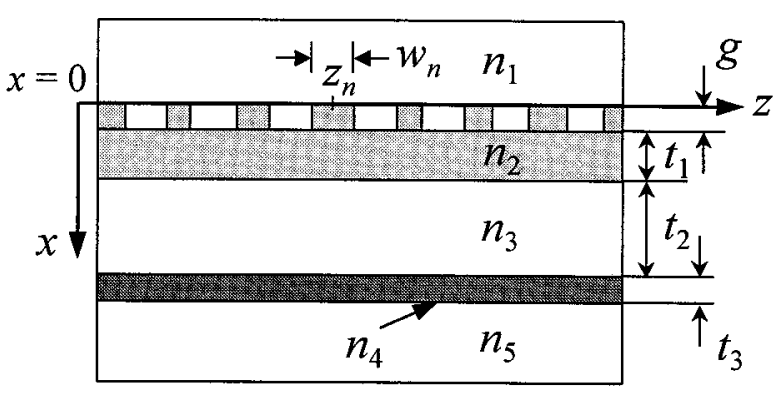

(a)

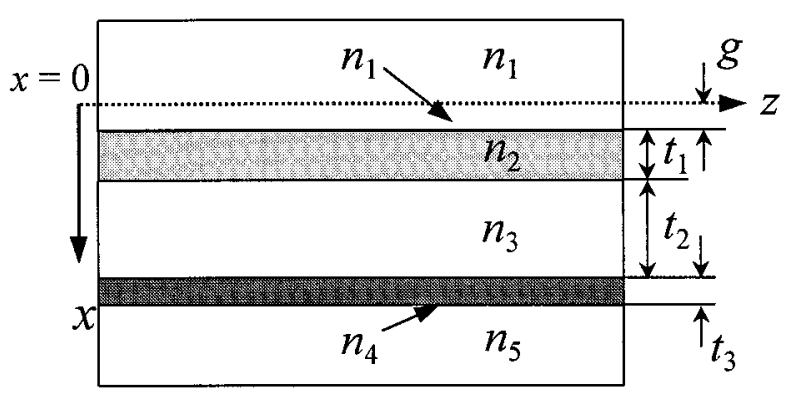

(b)

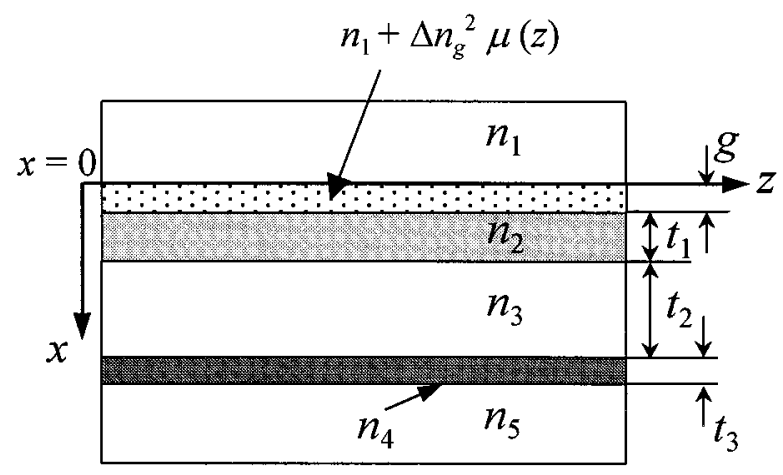

(c)

Fig. 1. (a) Waveguide structure and the refractive-index profile of a nonuniform vertical GACC with a varying grating width $w_{n}$ and varying positions $z_{n}$. (b) Reference waveguide structure and refractive-index profile for the GACC shown in (a). The original grating layer is taken to be the same as the cover layer, i.e., with a refractive index $n_{1}$. This configuration corresponds to a nonsynchronous codirectional coupler without a grating overlay. (c) Waveguide structure and the refractive-index profile for the local modes; this configuration represents a combination of the reference index profile and the zeroth-order index perturbation that is due to the nonuniformity of the duty ratios $\mu$.

present a systematic approach to analyzing the nonuniform vertical GACC's with a varying duty ratio and grating period. The coupled-local-mode formalism $^{12}$ is used here to analyze the nonuniform GACC's. This approach is used because the grating is located on one of the two waveguide layers, and the nonuniform grating duty ratio will change the average local refractive index of the grating layer such that the local guided modes will be different from point to point. Conventionally, a nonuniform grating is described by use of a complex taper function. The amplitude of the taper function corresponds to the varying coupling coefficient, and the phase characterizes the aperiodicity. ${ }^{13}$ Here the effective taper function of a nonuniform vertical GACC with a varying duty ratio and period is derived rigorously with the aid of the Poisson sum formula. ${ }^{14}$ This formula was originally adopted by Ishimaru ${ }^{15}$ in his treatment of nonuniform antenna arrays.

The contents of this paper are as follows: In Section 2 the functional form of the nonuniform rectangular grating is rewritten in a more intuitive way that is also suitable for the application of the Poisson sum formula. The nonuniform grating is then expanded into a quasi-Fourier series whose $m$ th-order expansion coefficient corresponds to the complex taper function of the $m$ th-order grating. The local grating parameters can be defined in an unambiguous way. Section 3 outlines the formalism of the coupled-local-mode theory. The zerothorder index perturbation of the quasi-Fourier series is used to define the local index profile from which we can solve the local array modes. These local modes are then regarded as unperturbed modes with respect to the almost-periodic grating, and a set of coupled-mode equations are derived. These coupled-mode equations are further transformed into a form known as the Zakharov-Shabat (ZS) system $^{10,16}$ such that the GLM inverse-scattering technique can be used to synthesize the grating filter. In Section 4, we demonstrate two examples of the GACC filter design by using the GLM method. One example is a third-order Butterworth filter; the other is a linear filter, or so-called power-discriminator filter in Ref. 9. Finally, we draw main conclusions in Section 5.

\section{Poisson Sum Formula for the Expansion of Index Perturbations}

The geometric structure of a typical almost-periodic vertical GACC with nonuniform duty ratios is shown schematically in Fig. 1(a). The transverse structure is composed of the following layers: a cover layer with a refractive index $n_{1}$, a grating layer with a thickness $g$ and alternating refractive indices $n_{1}$ and $n_{2}$, a top waveguide layer with a thickness $t_{1}$ and a refractive index $n_{2}$, a separation layer with a thickness $t_{2}$ and a refractive index $n_{3}$, a bottom waveguide layer with a thickness $t_{3}$ and a refractive index $n_{4}$, and the substrate with a refractive index $n_{5}$. The grating is rectangular in shape, and we denote the center longitudinal position of the $n$th rectangle with an index $n_{2}$ as $z_{n}$; the corresponding width of the rectangle is denoted as $w_{n}$ [see Fig. 1(a)]. We define a reference waveguide refractive-index profile $\bar{n}^{2}(x)$ as the one whose grating layer is the same as the cover layer, as shown in Fig. 1(b). This reference structure is just a nonsynchronous codirectional coupler without the grating overlay. Take this reference index profile as the unperturbed one; the index 
perturbation of a rectangular-shaped grating then can be described by use of

$$
\Delta n^{2}(x, z)= \begin{cases}\sum_{n=1}^{N} \Delta n_{g}{ }^{2} u\left(\frac{z-z_{n}}{w_{n}}\right) & 0<x<g \\ 0 & \text { elsewhere }\end{cases}
$$

where $\Delta n_{g}{ }^{2}=n_{2}{ }^{2}-n_{1}{ }^{2}$ and $u(z)$ is the unit-square function, which is unity when $|z| \leq 0.5$ and is zero elsewhere. The summation can be formally extended from $-\infty$ to $\infty$ if one assumes that $w_{n}=0$ when $n<1$ or $n>N$. The grating positions and widths are regarded as sampled values of two continuous functions, i.e., $z_{n}=z(n)$ and $w_{n}=w(n)$.

We next transform the summation into integrals by invoking the Poisson sum formula, which reads

$$
\sum_{n=-\infty}^{\infty} f(n)=\sum_{m=-\infty}^{\infty} \int_{-\infty}^{\infty} f(n) \exp (i 2 m \pi n) \mathrm{d} n
$$

where $f(n)$ is a continuous function of $n$. The transformed index perturbation becomes

$$
\Delta n^{2}(z)=\Delta n_{g}{ }^{2} \sum_{m=-\infty}^{\infty} \int_{-\infty}^{\infty} u\left[\frac{z-z(n)}{w(n)}\right] \exp [i 2 m \pi n] \mathrm{d} n .
$$

Note that henceforth the expression for $\Delta n^{2}(z)$ is valid within $0<x<g$ and zero outside this range. Because $z_{n}$ always increases with $n$, the position function $z(n)$ is a monotonically increasing function and is thus invertable. Let the inverse function be $n(z)$, which is called the source-number function. ${ }^{15}$ As a result of the assumption of almost periodicity, this function is assumed to have form

$$
n=n(z)=z / \Lambda^{(0)}+v(z),
$$

where $\Lambda^{(0)}$ is a reference period and $v(z)$ is a slowly varying function of $z$. Additionally, $v(z) \ll 1$ for an almost-periodic grating. The derivative $\mathrm{d} n / \mathrm{d} z$ is used in the expressions that follow, and it has the physical meaning of the inverse of the local period (note that $\mathrm{d} n / \mathrm{d} z \cong \Delta n / \Delta z_{n, n+1} \cong 1 / \Lambda_{n}$ ). Thus we define the local period function $\Lambda(z)$ as

$$
\frac{1}{\Lambda(z)} \equiv \frac{\mathrm{d} n}{\mathrm{~d} z}=\frac{1}{\Lambda^{(0)}}+\frac{\mathrm{d} v}{\mathrm{~d} z} .
$$

Now we change the integration variable from $n$ to $z^{\prime \prime}$ and obtain

$$
\Delta n^{2}(z)=\Delta n_{g}{ }^{2} \sum_{m=-\infty}^{\infty} \int_{-\infty}^{\infty} u\left[\frac{z-z^{\prime \prime}}{w\left(z^{\prime \prime}\right)}\right] \frac{\exp \left[i 2 m \pi n\left(z^{\prime \prime}\right)\right]}{\Lambda\left(z^{\prime \prime}\right)} \mathrm{d} z^{\prime \prime} .
$$

Note that the local period function $\Lambda\left(z^{\prime \prime}\right)$ in the integrand of Eq. (6) results from the factor $\mathrm{d} n / \mathrm{d} z^{\prime \prime}$ that is due to the change of integration variable. By further changing the integration variable by $z^{\prime \prime}=z-z^{\prime}$, we derive the quasi-Fourier series expansion for the index perturbation as

$$
\Delta n^{2}(z)=\sum_{m=-\infty}^{\infty} \Delta n_{m}{ }^{2}(z) \exp \left[\operatorname{im} \frac{2 \pi}{\Lambda^{(0)}} z\right]
$$

with the $m$ th-order expansion-coefficient function $\Delta n_{m}{ }^{2}(z)$ given by

$$
\begin{aligned}
\Delta n_{m}{ }^{2}(z)= & \Delta n_{g}{ }^{2} \int_{-\infty}^{\infty} u\left[\frac{z^{\prime}}{w\left(z-z^{\prime}\right)}\right] \\
& \times \frac{\exp \left[i 2 m \pi v\left(z-z^{\prime}\right)\right]}{\Lambda\left(z-z^{\prime}\right)} \\
& \times \exp \left\{-i m\left[2 \pi / \Lambda^{(0)}\right] z^{\prime}\right\} \mathrm{d} z^{\prime},
\end{aligned}
$$

where we used expression (4) for $n(z)$.

The expansion in Eq. (8) is exact so far, and we proved that the index perturbation of a slowly varying nonuniform almost-periodic grating can be expanded as a quasi-Fourier series. The expansion coefficients are also slowly varying functions of $z$ and act as the effective taper function of the corresponding grating order. We can further simplify Eq. (8) by noting that the integration interval for $z^{\prime}$ is approximately limited within $\pm \bar{w} / 2$, where $\bar{w}$ is some average value of the grating width. Because the grating is assumed to be slowly varying and if the conditions

$$
\mathrm{d} w / \mathrm{d} z \ll 1, \quad \mathrm{~d} \Lambda / \mathrm{d} z \ll 1
$$

are satisfied, we can approximate functions (9) with their zeroth-order expansion, i.e., $w\left(z-z^{\prime}\right) \cong w(z)$ and $\Lambda\left(z-z^{\prime}\right) \cong \Lambda(z)$, respectively. However, the function $v\left(z-z^{\prime}\right)$ in the exponential must be consistently approximated by use of the first-order expansion because its first derivative is related to the local period function as

$$
v\left(z-z^{\prime}\right) \cong v(z)-\frac{\mathrm{d} v}{\mathrm{~d} z} z^{\prime}=v(z)+\frac{z^{\prime}}{\Lambda^{(0)}}-\frac{z^{\prime}}{\Lambda(z)} .
$$

Substituting these approximations into Eq. (8), we obtain a simplified expression for the expansioncoefficient function:

$$
\begin{aligned}
\Delta n_{m}{ }^{2}(z) \cong & \Delta n_{g}{ }^{2} \frac{\exp [i 2 m \pi v(z)]}{\Lambda(z)} \int_{-\infty}^{\infty} u\left[\frac{z^{\prime}}{w(z)}\right] \\
& \times \exp \left\{-i m[2 \pi / \Lambda(z)] z^{\prime}\right\} \mathrm{d} z^{\prime}
\end{aligned}
$$

With expression (11) the quasi-Fourier expansion can be rewritten as

$$
\Delta n^{2}(z)=\sum_{m=-\infty}^{\infty} \Delta \hat{n}_{m}{ }^{2}(z) \exp \left[i m \int_{0}^{z} \frac{2 \pi}{\Lambda\left(z^{\prime}\right)} \mathrm{d} z^{\prime}\right] .
$$

Note that expression (12) is expanded by use of the local grating period $\Lambda(z)$ in integral form but not the reference grating period $\Lambda^{(0)}$. And the newly defined expansion coefficients are 


$$
\begin{aligned}
\Delta \hat{n}_{m}{ }^{2}(z) & \equiv \Delta{n_{m}}^{2}(z) \exp [-i 2 m \pi v(z)] \\
& =\frac{\Delta n_{g}{ }^{2}}{\Lambda(z)} \int_{-\infty}^{\infty} u\left[\frac{z^{\prime}}{w(z)}\right] \exp \left\{-i m[2 \pi / \Lambda(z)] z^{\prime}\right\} \mathrm{d} z^{\prime} .
\end{aligned}
$$

Expression (13) is the usual formula for evaluating the Fourier coefficients of a unit-squared function $u(z)$. However, the local parameters $\Lambda(z)$ and $w(z)$ are used instead. For a slowly varying, almostperiodic grating this approach seems intuitively true; however, we gave a rigorous derivation here. Using the definition of the unit-squared function allows the $m$ th-order coefficient to be evaluated readily as

$$
\Delta \hat{n}_{m}{ }^{2}(z)=\left\{\begin{array}{ll}
\Delta n_{g}{ }^{2} \mu(z) & m=0 \\
\frac{\Delta n_{g}{ }^{2}}{m \pi} \sin [m \pi \mu(z)] & m \neq 0
\end{array},\right.
$$

where we defined the local duty ratio $\mu(z)$ as

$$
\mu(z)=\frac{w(z)}{\Lambda(z)} .
$$

Equations (14) and (15) are used in the following analysis and design of nonuniform GACC filters. And, without a loss of generality, we mainly consider couplings caused by the first-order grating, i.e., $m=$ 1.

\section{Coupled-Local-Mode Theory for Nonuniform Grating-Assisted Codirectional Couplers}

In this section a coupled-local-mode theory is proposed that is based on the quasi-Fourier series expansion developed in Section 2 for the analysis of a nonuniform GACC with a varying duty ratio and period. Consider the reference refractive-index profile $\bar{n}^{2}(x)$ that is shown in Fig. 1(b). The coordinate system is also shown in Fig. 1. As was mentioned above, as the duty ratio is varied along the grating, the local guiding structure is changed accordingly. Here the zeroth-order expansion of the grating is used to define the local index profile as

$$
\begin{aligned}
& n_{0}{ }^{2}(x, z) \\
& \left\{\begin{array}{cc}
\bar{n}^{2}(x)+\Delta n_{0}{ }^{2}(z)=n_{1}{ }^{2}+\Delta n_{g}{ }^{2} \mu(z) & 0<x<g \\
\bar{n}^{2}(x) & \text { elsewhere }
\end{array}\right.
\end{aligned}
$$

where we used Eq. (14) for $\Delta n_{0}{ }^{2}(z)$. The index profile of the local waveguide structure is shown schematically in Fig. 1(c). Note that the squared refractive index of the grating layer is linearly dependent on the local duty ratio: it equals $n_{1}{ }^{2}$ when $\mu=$ 0 and ${n_{2}}^{2}$ when $\mu=1$. In the following we consider only couplings between the fundamental TE modes.

The vector electric and magnetic fields of the local fundamental modes can be expressed as

$$
\mathbf{E}_{j}=\mathbf{e}_{j}\left[x, \beta_{j}(z)\right] \exp \left[i \int_{0}^{z} \beta_{j}\left(z^{\prime}\right) \mathrm{d} z^{\prime}\right],
$$

$$
\mathbf{H}_{j}=\mathbf{h}_{j}\left[x, \beta_{j}(z)\right] \exp \left[i \int_{0}^{z} \beta_{j}\left(z^{\prime}\right) \mathrm{d} z^{\prime}\right],
$$

respectively, where $j=1$ or $j=2$ for the local even and odd array modes, respectively. For TE modes, the electric fields are $y$ polarized, and the $x$ component of the magnetic field is zero in our coordinate system, i.e., $\mathbf{e}_{j}=\mathbf{y} e_{y j}$ and $h_{x j}=0$. The local mode fields are $z$ dependent through the dependence on $\beta_{j}(z)$, which is, in turn, $z$ dependent through the dependence on $\mu(z)$. The local mode fields satisfy the following wave equations:

$$
\begin{aligned}
\left\{\frac{\mathrm{d}^{2}}{\mathrm{~d} x^{2}}+k_{0}^{2}\left[\bar{n}^{2}(x)+\Delta \hat{n}_{0}^{2}(x, z)\right]\right\} e_{y j} & =\beta_{j}^{2}(z) e_{y j}, \\
h_{x j} & =-\frac{\beta_{j}}{\omega \mu} e_{y j}, \\
h_{z j} & =\frac{-i}{\omega \mu} \frac{\mathrm{d} e_{y j}}{\mathrm{~d} x},
\end{aligned}
$$

with the appropriate boundary conditions. In Eqs. (18), $k_{0}=2 \pi / \lambda$ is the vacuum wave number, $\omega$ is the frequency, and $\mu_{0}$ is the permeability in free space. Because we consider only the codirectional couplings, the total electric field can be expressed in terms of the local even and odd modes as

$$
\mathbf{E}_{t}=\sum_{j=1,2} a_{j}(z) \mathbf{e}_{j}\left[x, \beta_{j}(z)\right] \exp \left[i \int_{0}^{z} \beta_{j}\left(z^{\prime}\right) \mathrm{d} z^{\prime}\right],
$$

where $a_{j}(z)$ is the slowly varying amplitude of mode $j$. As was mentioned above, we consider couplings caused by the first-order grating, $m=1$, i.e., $\Delta \hat{n}_{1}{ }^{2}(z)$. Substituting Eq. (19) into Maxwell's equations, as outlined in Ref. 12, with the first-order grating perturbation $\Delta \hat{n}_{1}{ }^{2}(z)$ taken into consideration allows the coupled-local-mode equations to be derived as

$$
\begin{aligned}
& \frac{\mathrm{d} a_{1}}{\mathrm{~d} z}=C_{11} a_{1}+C_{12} a_{2} \exp \left\{i \int_{0}^{z}\left[\beta_{2}\left(z^{\prime}\right)-\beta_{1}\left(z^{\prime}\right)\right] \mathrm{d} z^{\prime}\right\}, \\
& \frac{\mathrm{d} a_{2}}{\mathrm{~d} z}=C_{22} a_{2}+C_{21} a_{1} \exp \left\{i \int_{0}^{z}\left[\beta_{1}\left(z^{\prime}\right)-\beta_{2}\left(z^{\prime}\right)\right] \mathrm{d} z^{\prime}\right\},
\end{aligned}
$$

where the coupling coefficients are given by

$$
\begin{aligned}
C_{j k}= & \frac{1}{4} \int_{-\infty}^{\infty}\left[h_{x j} \frac{\partial e_{y k}}{\partial z}-e_{y j} \frac{\partial h_{x k}}{\partial z}\right] \mathrm{d} x \\
& +i \frac{\omega \epsilon_{0}}{4} \exp \left[i \int_{0}^{z} \frac{2 \pi}{\Lambda\left(z^{\prime}\right)} \mathrm{d} z^{\prime}\right] \int_{0}^{g} \Delta \hat{n}_{1}{ }^{2} e_{y j} e_{y k} \mathrm{~d} x .
\end{aligned}
$$


In deriving the expressions (20) and (21) the following orthogonality relations are used:

$$
\frac{1}{2} \int_{-\infty}^{\infty} \mathbf{z}\left(\mathbf{e}_{t j} \times \mathbf{h}_{t k}\right) \mathrm{d} x=\delta_{j k} .
$$

Note that there are two contributions to the coupling coefficients. The first term on the right-hand side of Eq. (21) comes from variations of the local mode fields. For $j=k$, this term vanishes because it is proportional to the $z$ derivative of the normalized power in Eq. (22). The second term on the righthand side of Eq. (21) represents couplings of the local modes through the first-order grating. By keeping only the phase-matching terms, we obtain

$$
\begin{aligned}
& \frac{\mathrm{d} a_{1}}{\mathrm{~d} z}=i \kappa(z) a_{2} \exp \left[-2 i \int_{0}^{z} \delta\left(z^{\prime}\right) \mathrm{d} z^{\prime}\right], \\
& \frac{\mathrm{d} a_{2}}{\mathrm{~d} z}=i \kappa(z) a_{1} \exp \left[2 \mathrm{i} \int_{0}^{z} \delta\left(\mathrm{z}^{\prime}\right) \mathrm{d} z^{\prime}\right],
\end{aligned}
$$

where we defined the local detuning parameter $\delta(z)$ as

$$
\delta(z)=\frac{1}{2}\left[\beta_{1}(z)-\beta_{2}(z)-\frac{2 \pi}{\Lambda(z)}\right]
$$

and the coupling coefficient $\kappa(z)$ as

$$
\kappa(z)=\Delta n_{g}{ }^{2} \frac{\omega \epsilon_{0}}{4 \pi} \sin [\pi \mu(z)] \int_{0}^{g} e_{y 1}[\mu(z)] e_{y 2}[\mu(z)] \mathrm{d} x .
$$

Note that the electric fields $e_{y j}$ used to evaluate the overlapping integral are also dependent on the local duty ratio $\mu(z)$. With transformations of the mode amplitudes by

$$
\begin{aligned}
& \tilde{a}_{1}(z)=a_{1}(z) \exp \left[i \int_{0}^{z} \delta\left(z^{\prime}\right) \mathrm{d} z^{\prime}\right], \\
& \tilde{a}_{2}(z)=a_{2}(z) \exp \left[-i \int_{0}^{z} \delta\left(z^{\prime}\right) \mathrm{d} z^{\prime}\right],
\end{aligned}
$$

Eqs. (23) become

$$
\begin{aligned}
& \mathrm{d} \tilde{a}_{1} / \mathrm{d} z=i \delta(z) \tilde{a}_{1}+i \kappa(z) \tilde{a}_{2}, \\
& \mathrm{~d} \tilde{a}_{2} / \mathrm{d} z=-i \delta(z) \tilde{a}_{2}+i \kappa(z) \tilde{a}_{1},
\end{aligned}
$$

which are the well-known coupled-wave equations for codirectional two-mode couplings. ${ }^{17}$ However, the detuning parameter as well as the coupling coefficient are both dependent on the longitudinal distance $z$.

To use the well-developed GLM inverse-scattering method for the synthesis of GACC filters requires that the above set of coupled-mode equations (27) be further transformed into a form known as the ZS system, ${ }^{10,16}$ and many methods have been developed to solve the inverse (synthesis) problems of the $\mathrm{ZS}$ system. We first define $\beta_{1}^{(0)}$ and $\beta_{2}^{(0)}$ as two reference propagation constants that correspond to the reference waveguide structure. The local propagation constants are expressed as $\beta_{j}(\lambda, z)=\beta_{j}^{(0)}(\lambda)+\Delta \beta_{j}(z$, $\lambda)$, where $j=1,2$. Let us define the following $z$-independent detuning parameter:

$$
\xi=\frac{1}{2}\left[\beta_{1}^{(0)}(\lambda)-\beta_{2}^{(0)}(\lambda)-\frac{2 \pi}{\Lambda^{(0)}}\right] .
$$

Equation (28) is obviously a function of the wavelength $\lambda$. The reference grating period $\Lambda^{(0)}$ is chosen such that $\xi\left(\lambda_{0}\right)=0$ for a given center wavelength $\lambda_{0}$.

We now make the following variable transformations:

$$
\begin{aligned}
& \nu_{1}(z)=a_{1}(z) \exp (i \xi z), \\
& \nu_{2}(z)=a_{2}(z) \exp (-i \xi z) .
\end{aligned}
$$

Then the original coupled equations (23) are transformed into the ZS system as

$$
\begin{aligned}
& \mathrm{d} \nu_{1}(z, \xi) / \mathrm{d} z-i \xi \nu_{1}(z, \xi)=q(z) \nu_{2}(z, \xi), \\
& \mathrm{d} \nu_{2}(z, \xi) / \mathrm{d} z+i \xi \nu_{2}(z, \xi)=-q^{*}(z) \nu_{1}(z, \xi),
\end{aligned}
$$

respectively, where the expression for the complex coupling potential $q(z)$ is

$$
q(z)=i \kappa(z) \exp [i \theta(z)],
$$

with $\kappa(z)$ given in Eq. (25) and the following expression for $\theta(z)$ :

$$
\theta(z)=2 \pi v(z)-\int_{0}^{z}\left[\Delta \beta_{1}\left(z^{\prime}\right)-\Delta \beta_{2}\left(z^{\prime}\right)\right] \mathrm{d} z^{\prime} .
$$

Equations (31) and (32) relate the complex coupling potential $q(z)$ to the local duty ratio $\mu(z)$ through the coupling coefficient $\kappa(z)$ and to the source-number function through $v(z)$. Having determined the source-number function $n(z)$, we can use it to find the corresponding positions $z_{n}$ and widths $w_{n}$ of the rectangular grating. This relation indicates that we might properly adjust these parameters, $w_{n}$ and $z_{n}$, to realize a coupling potential that results in a specific grating response.

\section{Design Examples}

We now present two vertical GACC filter design examples. The designs are based on the GLM inversescattering technique. Details of the GLM method can be found in Refs. 10 and 11 .

To apply the GLM inverse-scattering method, we must specify the scattering data of the ZS equation, and, in the forward-coupling case, one must specify the scattering coefficient ${ }^{10,11}$ as

$$
r(\xi)=v_{2}(L, \xi) / \nu_{1}(L, \xi),
$$

where $L$ is the interaction length of the grating. Basically, the GLM equation is a set of integral equations for solving the unknown coupling potential $q(z)$, provided the scattering coefficient $r(\xi)$ is given. 


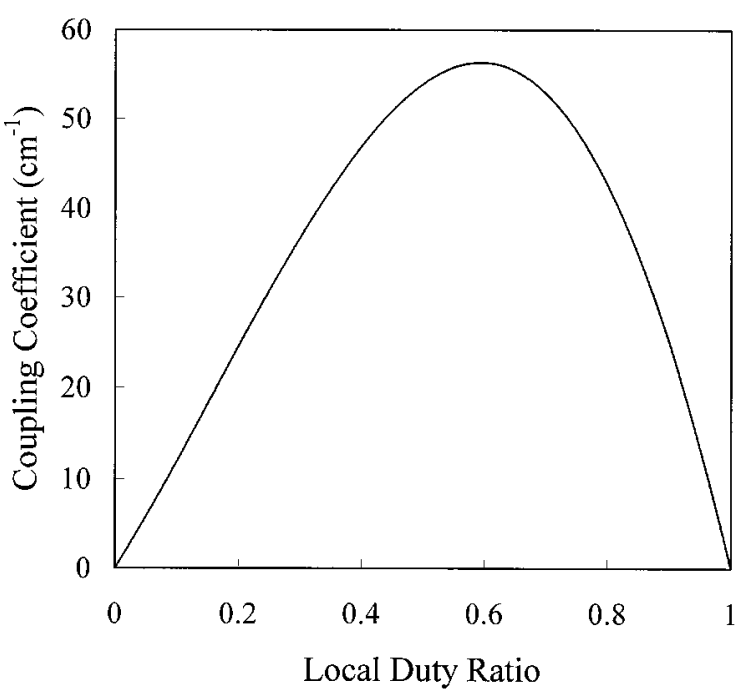

Fig. 2. Coupling coefficient $\kappa$ plotted as a function of the local duty ratio $\mu$ for a first-order $(m=1)$ grating.

Song and Shin ${ }^{10}$ showed that the GLM integral equations can be transformed into a set of linear equations for $q(z)$ and thus can be solved exactly if $r(\xi)$ is a rational function of $\xi$. We use this method in the following synthesis problem.

After the synthesized coupling potential $q(z)$ is obtained its amplitude can be used to determine the local duty ratio $\mu(z)$ through Eq. (25). After the local duty ratio is known the local propagation constants can be solved by use of Eq. (18). And from the phase of $q(z)$ the local grating period can be derived by the differentiation of Eq. (32) with respect to $z$ :

$$
\Lambda(z)=\left\{\frac{1}{\Lambda^{(0)}}+\frac{1}{2 \pi} \frac{\mathrm{d} \theta}{\mathrm{d} z}+\frac{\left[\Delta \beta_{1}(z)-\Delta \beta_{2}(z)\right]}{2 \pi}\right\}^{-1} .
$$

Note that there are three contributions to the local period. The first term in braces in Eq. (34) is the constant reference grating period $\Lambda^{(0)}$. The second term comes from the required grating phase. And the third compensates for the effective chirp that is caused by the nonuniform local propagation constants. Finally, the local grating width is given by $w(z)=\mu(z) \Lambda(z)$.

The material parameters used in the following design were as follows: The cover layer, the separation layer, and the substrate are all composed of InP. The thickness of the separation layer is $t_{2}=1 \mu \mathrm{m}$. The top waveguide layer is InGaAsP latticed matched to InP with a bandgap wavelength of $\lambda_{g}=$ $1.45 \mu \mathrm{m}$ and a thickness of $t_{1}=0.5 \mu \mathrm{m}$. The bottom waveguide layer is also InGaAsP latticed matched to InP with a bandgap wavelength of $\lambda_{g}=1.1 \mu \mathrm{m}$ and a thickness of $t_{3}=0.3 \mu \mathrm{m}$. Material dispersion of InGaAsP latticed matched to the InP substrate is taken into consideration by use of the refractiveindex model of Ref. 18.

In Fig. 2, we show the dependence of the coupling coefficient on the local duty ratio for a first-order grating $(m=1)$. The thickness of the grating layer is $g=0.07 \mu \mathrm{m}$. Note from the figure that, apart from the explicit $\sin (\pi \mu)$ dependence (which is obviously symmetric to $\mu$ ), the coupling coefficient $\kappa$ also depends on the local duty ratio through the overlapping integral of the two local mode fields [see Eq. (25)].

In the case of forward coupling, instead of the scattering coefficient, we often specify the power transfer function, which is defined as

$$
T(\xi)=\left|\frac{\nu_{2}(L, \xi)}{\nu_{1}(0, \xi)}\right|^{2} .
$$

As a result of power conservation, $\left|\nu_{2}(L, \xi)\right|^{2}+\mid \nu_{1}(L$, $\xi)\left.\right|^{2}=\left|v_{1}(0, \xi)\right|^{2}$, function (35) can be related to the scattering coefficient $r(\xi)$ as follows:

$$
|r(\xi)|^{2}=\frac{T(\xi)}{1-T(\xi)} .
$$

We can use relation (36) to derive the required scattering-coefficient function. In the first example, we consider a third-order Butterworth filter whose frequency response is defined as

$$
T(\xi)=\frac{T_{\max }}{1+\left(\xi / \xi_{c}\right)^{6}},
$$

where $T_{\max }$ is the maximum power transfer and $\xi_{c}$ is the filter half-power point. By substituting Eq. (37) into Eq. (36) and assuming that the scattering coefficient satisfies $r^{*}(\xi)=r(-\xi)$, such that $|r(\xi)|^{2}=$ $r(\xi) r(-\xi)$, we obtain a rational representation for $r(\xi)$ as

$$
r(\xi)=\frac{N(\xi)}{D(\xi)}
$$

with

$$
\begin{aligned}
& N(\xi)=\left(T_{\max }\right)^{1 / 2}, \\
& D(\xi)=\left(1-T_{\max }\right)^{1 / 2}+i\left(\xi / \xi_{c}\right)^{3} .
\end{aligned}
$$

Figure 3 shows the synthesized filter response, which indicates that a good result is obtained in this case. The maximum power transfer is $T_{\max }=0.9 \mathrm{in}$ this case. Because $\Lambda^{(0)}$ is chosen such that $\xi\left(\lambda_{0}\right)=0$, for small deviations from the center wavelength $\lambda_{0}$ the detuning parameter $\xi$ is proportional to the wavelength deviation as

$$
\xi \cong \frac{1}{2}\left[\frac{\mathrm{d} \beta_{1}^{(0)}}{\mathrm{d} \lambda}-\frac{\mathrm{d} \beta_{2}^{(0)}}{\mathrm{d} \lambda}\right]_{\lambda=\lambda 0}\left(\lambda-\lambda_{0}\right)
$$

where the center wavelength $\lambda_{0}$ is chosen to be 1550 $\mathrm{nm}$, and the half-power detuning parameter is $\xi_{c}=$ $1.21 \mathrm{~mm}^{-1}$. The corresponding FWHM wavelength range is approximately $2.5 \mathrm{~nm}$, as can be seen from Fig. 3.

The reconstructed grating's local width and local period are shown in Figs. 4 and 5, respectively. Note that a sign change of the grating width $w(z)$ indicates the insertion of a $\pi$ phase shift at the crossing point. 


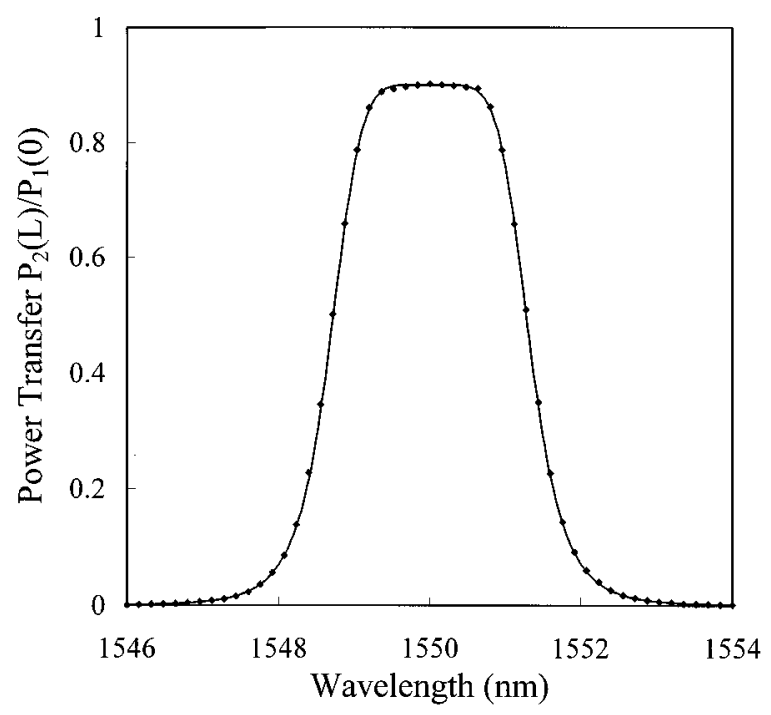

Fig. 3. Synthesized filter response (points) compared with the desired filter response (solid curve) of a third-order Butterworth GACC filter designed by use of the coupled-local-mode equations and the GLM technique.

Because a symmetric filter response such as with the Butterworth filter will result in a real-valued coupling potential, $\theta(z)$ is either zero or $\pi \cdot{ }^{19}$ However, as was mentioned above, the grating period varies in such a way as to compensate for the effective chirp that is caused by the variation of the differential local propagation constants [see Eq. (33) and Fig. 5].

As a second example, we consider a linear filter with a filter response of

$$
T(\xi)= \begin{cases}T_{\max }\left(1-\xi / \xi_{c}\right) / 2 & \left|\xi / \xi_{c}\right| \leq 1 \\ 0 & \text { elsewhere }\end{cases}
$$

where we choose a value of $T_{\max }=0.7$.

Given the above power transfer function, we can find the corresponding $r(\xi)$ with the aid of Eq. (36).

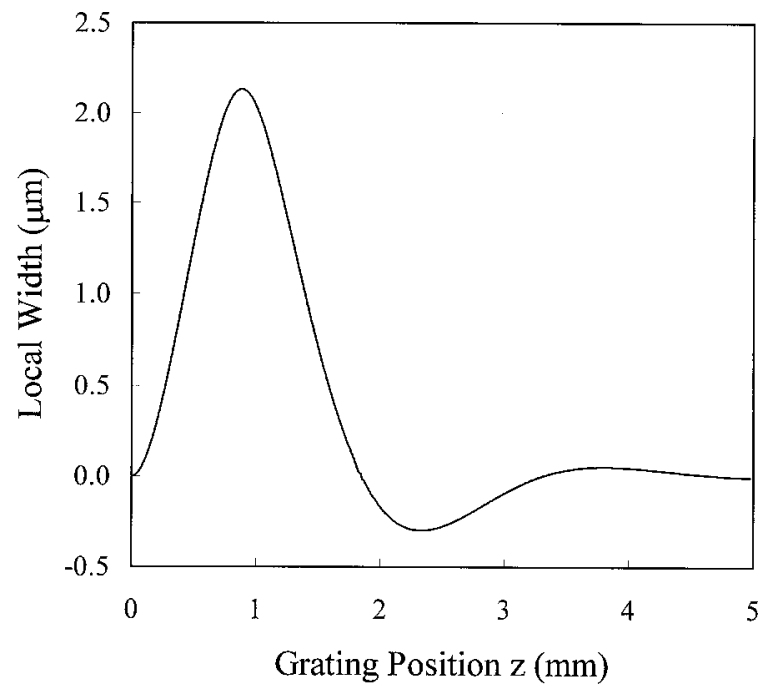

Fig. 4. Local grating width $w$ plotted as a function of the grating position $z$ for a third-order Butterworth GACC filter.

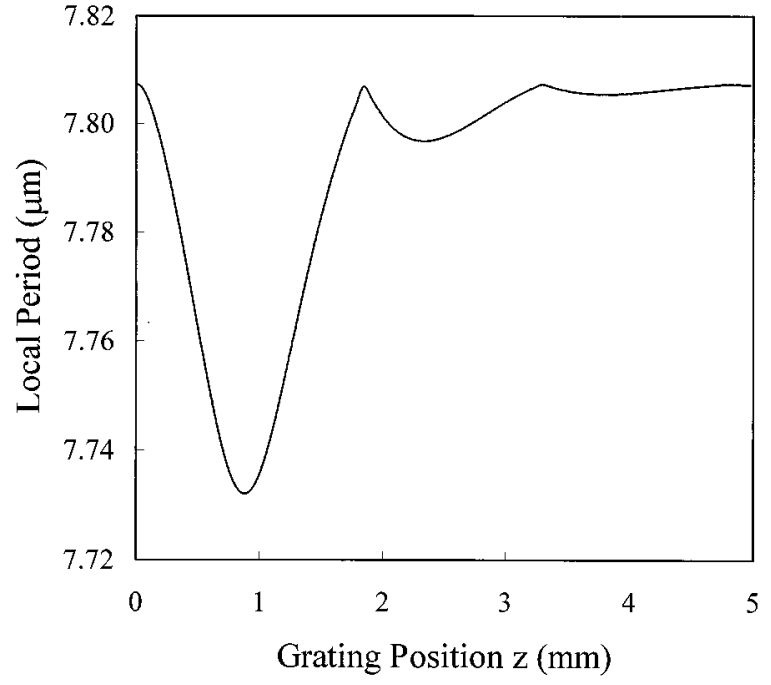

Fig. 5. Local grating period $\Lambda$ plotted as a function of the grating position $z$ for a third-order Butterworth GACC filter.

In this case there are no analytic expressions for $N(\xi)$ and $D(\xi)$. Thus we use a fifth-order, polynomial, least-squares fit to derive $N(\xi)$ and a sixth-order, polynomial, least-squares fit to derive $D(\xi)$. In fitting the linear filter's scattering coefficient $r(\xi)$, a sixth-order Butterworth filter is included in the denominator of function $T(\xi)$ [Eq. (41)] to derive the transmission to zero for wavelengths outside the specified region.

Figure 6 shows the synthesized linear filter response. The desired linear wavelength region is from 1548 to $1552 \mathrm{~nm}$. Because the GLM method is exact, as described above, the discrepancy between the synthesized spectrum and the desired one comes mainly from the fitting error of the rational function

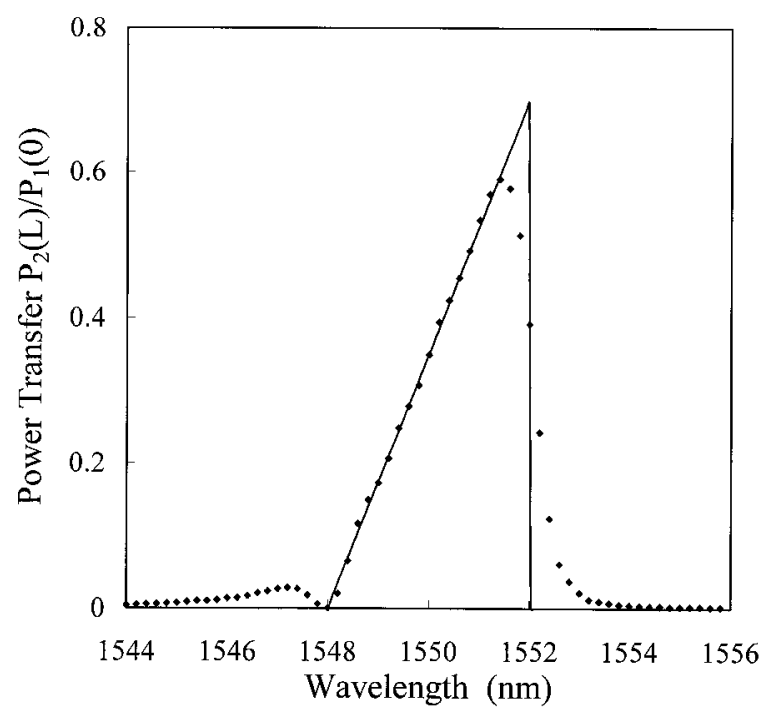

Fig. 6. Response of the linear GACC filter designed by use of the coupled-local-mode equations and the GLM technique: the synthesized response (points) compared with the desired response (solid curve). 


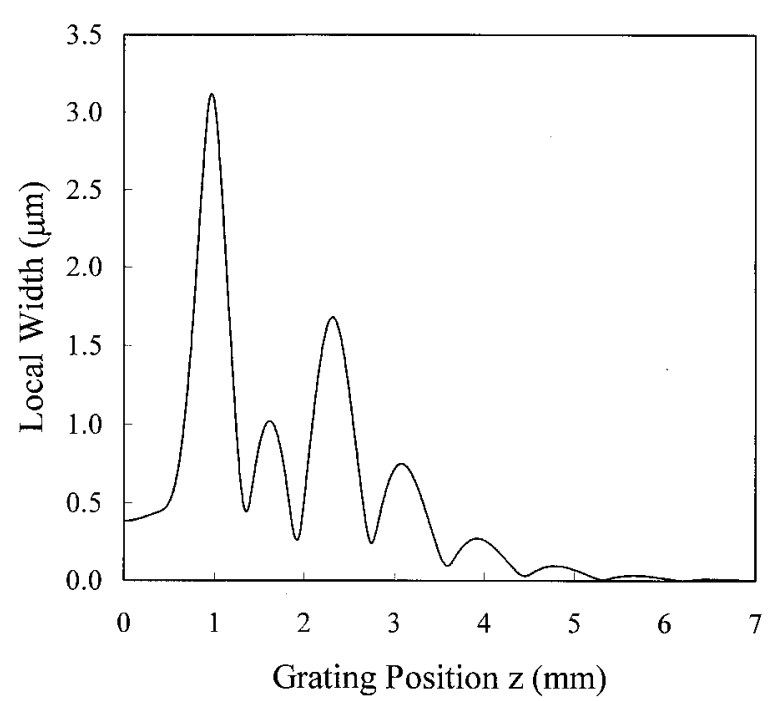

Fig. 7. Local grating width $w$ plotted as a function of the grating position $z$ for a linear GACC filter.

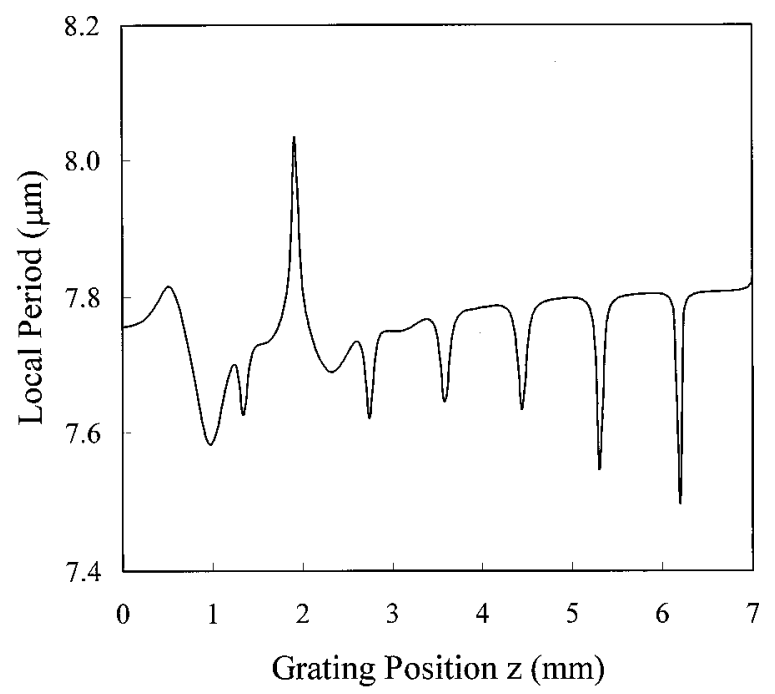

Fig. 8. Local grating period $\Lambda$ plotted as a function of the grating position $z$ for a linear GACC filter.

to $r(\xi)$. Figures 7 and 8 show the grating's local width and local period as functions of the longitudinal distance $z$. The designed total filter length is approximately $7 \mathrm{~mm}$. Qualitatively, the narrower the filter bandwidth, the longer the interaction length required.

\section{Conclusions}

In summary, an approach based on the Poisson sum formula and coupled-local-mode theory has been presented for the analysis of almost-periodic GACC filters with nonuniform duty ratios. A rectangular grating with nonuniform widths and variable spacings is expanded into a quasi-Fourier series by use of the Poisson sum formula. Without a loss of generality, we consider the first-order grating and the couplings between the two fundamental TE array modes.
On the basis of the quasi-Fourier series expansion the zeroth-order refractive-index perturbation combined with the $z$-independent reference waveguide structure has been used to define the local refractiveindex profile from which we derive the local mode fields and the local propagation constants. These local modes act as unperturbed modes with respect to the first-order grating, and a set of coupled-localmode equations was then used to analyze the interaction of these local modes. We have also pointed out that the approach that uses the Poisson sum formula to define clearly the local grating parameters is very suitable for combination with the coupledlocal-mode theory for the analysis of nonuniform GACC filters.

By transforming the coupled-local-mode equations into the ZS system, we have applied the well-known GLM inverse-scattering method to the design of special GACC filters. The GLM integral equations can be solved exactly, provided the scattering data are expressed as a rational function of the wavelengthdetuning parameter and the accuracy of the GLM method is limited by the fitting error of the rational function.

The design has been illustrated with two examples: One is a third-order Butterworth filter, and the other is a linear filter. For these synthesized GACC filters the grating widths and periods are larger than the contradirectional ones and may be realized by use of conventional photolithography or $e$-beam lithography techniques.

The authors are grateful for the support in part by the National Science Council Taiwan under contract NSC 89-2215-E-002-013 and by the Education Ministry, Taiwan, under contract 89-E-FA06-2-4.

\section{References}

1. D. Marcuse, "Directional couplers made of nonidentical asymmetric slabs. Part II: grating-assisted couplers," J. Lightwave Technol. 5, 268-273 (1987).

2. I. Kim, R. C. Alferness, L. L. Buhl, U. Koren, B. I. Miller, M. G. Young, M. A. Newkirk, M. D. Chien, T. L. Koch, G. Raybon, and C. A. Burrus, "InGaAs/InGaAsP MQW optical amplifier integrated with grating-assisted vertical-coupler noise filter," IEEE Photon. Technol. Lett. 5, 1319-1321 (1993).

3. W. Vanderbauwhede, I. Moerman, P. Van Daele, and P. Demeester, "Wavelength tuning by quantum well electrorefraction in a grating-assisted vertical coupler filter with InGaAsP/ InAlGaAs MQW waveguide," Opt. Quantum Electron. 28, 583590 (1996).

4. H. Sakata, "Analysis of wavelength-selective photodetectors based on grating-assisted forward coupling," J. Lightwave Technol. 11, 560-566 (1993).

5. Y. H. Jan, G. A. Fish, L. A. Coldren, and S. P. DenBaars, "Demonstration of InP-InGaAsP vertical grating-assisted codirectional coupler filters and receivers with tapered coupling coefficient distributions," IEEE Photon. Technol. Lett. 9, 994996 (1997).

6. R. C. Alferness and P. S. Cross, "Filter characteristics of codirectionally coupled waveguides with weighted coupling," IEEE J. Quantum Electron. 14, 843-847 (1978).

7. H. Sakata, "Sidelobe suppression in grating-assisted wavelength-selective couplers,” Opt. Lett. 17, 463-465 (1992).

8. M. Yamada and K. Sakuda, "Analysis of almost-periodic dis- 
tributed feedback slab waveguides via a fundamental matrix approach," Appl. Opt. 26, 3474-3478 (1987).

9. M. Matsuhara, K. O. Hill, and A. Watanabe, "Optical-waveguide filters: synthesis,” J. Opt. Soc. Am. 65, 804-809 (1975).

10. G. H. Song and S. Y. Shin, "Design of corrugated waveguide filters by the Gel'fand-Levitan-Marchenko inverse-scattering method," J. Opt. Soc. Am. A 2, 1905-1915 (1985).

11. K. A. Winick, "Design of grating-assisted waveguide couplers with weighted coupling," J. Lightwave Technol. 9, 1481-1492 (1991).

12. A. W. Snyder and J. D. Love, Optical Waveguide Theory (Elsevier, New York, 1969).

13. H. Kogelnik, "Filter response of nonuniform almost-periodic structures," Bell Syst. Tech. J. 48, 109-126 (1976).

14. P. N. Morse and H. Feshbach, Methods of Theoretical Physics (McGraw-Hill, New York, 1953), p. 466.
15. A. Ishimaru, "Theory of unequally spaced arrays," IRE Trans. Antennas Propag. 10, 691-702 (1963).

16. V. E. Zakharov and A. B. Shabat, "Exact theory of twodimensional self-focusing and one-dimensional selfmodulation of waves in nonlinear media," Sov. Phys. JETP 34, 62-69 (1972).

17. H. Kogelnik, "Theory of dielectric waveguides," in Integrated Optics, T. Tamir, ed. (Springer-Verlag, New York, 1975), Chap. 2.

18. C. H. Henry, L. F. Johnson, R. A. Logan, and D. P. Clarke, "Determination of the refractive index of InGaAsP epitaxial layers by mode line luminescence spectroscopy," IEEE J. Quantum Electron. 21, 1887-1892 (1985).

19. G. H. Song, "Theory of symmetry in optical filter responses," J. Opt. Soc. Am. A 11, 2027-2037 (1994). 